characteristics of incident and prevalent cancer cases in the CRN is essential for investigators to evaluate the feasibility of CRN research projects Methods: Characteristics of health plan enrollees and prevalent (i.e., history of) and incident cancers were collected across the 9 funded CRN sites. Data were obtained from the CRN Cancer Counter - a tool to aggregate patient counts across sites from centralized summary data-as well as a centrallydeveloped SAS ${ }^{\circ}$ program run against each site's Virtual Data Warehouse. Descriptive analyses focused on distributions of malignant cancer incidence and prevalence by age and anatomic site as well as by length of enrollment before and after diagnosis. Results: As of mid-2013, more than 9 million persons were enrolled in CRN site health plans. Of those enrollees, more than half a million were diagnosed with cancer during or before each site's last available year of cancer incidence data (2008-2011, varied by site). Prostate, breast, non-melanoma skin, and colon cancers had the highest prevalence and incidence. Overall, 22\% of survivors were diagnosed prior to 1998 Examination of enrollees at one CRN site with a diagnosis of cancer in 2011 found that nearly half had been enrolled in the health plan for at least 15 years prior to diagnosis. Enrollment for cancer patients will be examined at additional CRN sites. Conclusions: The CRN is an excellent setting for prospective and retrospective studies of cancer etiology, screening, treatment and outcomes. Long periods of enrollment prior to cancer diagnosis facilitate retrospective studies of cancer etiology. Large numbers of cancer survivors with low disenrollment rates facilitate prospective observational studies and trials focused on cancer outcomes.

Keywords: Cancer Research Network; Epidemiology doi:10.3121/cmr.2014.1250.ps2-38

PS2-39:

Depression and Its Relationship to Perceived Financial Burden among Long-term Rectal Cancer Survivors with Ostomies and Anastomoses

Yuda Chongpison ${ }^{1}$; Mark Hornbrook ${ }^{2}$; Robin Harris ${ }^{1}$; Marcia Grant ${ }^{3}$; Joanna Bulkley $^{2}$; Christopher Wendel ${ }^{4}$; Lisa Herrinton ${ }^{5}$; Robert Krouse ${ }^{1}$

${ }^{1}$ University of Arizona; ${ }^{2}$ Kaiser Permanente Northwest; ${ }^{3}$ City of Hope National Medical Center; ${ }^{4}$ Southern Arizona Veterans Affairs Health Care System; ${ }^{5}$ Kaiser Permanente Northern California

Background/Aims: Consequences of surgery for rectal cancer (permanent ostomy (PO), anastomosis (AN), or temporary ostomy (TO) followed by anastomosis) can affect survivors' psychological and financial well-being. We investigated depression and its association with perceived financial burden resulting from their illness/treatment among long-term ( $\geq 5$ years post-diagnosis) survivors. Methods: A mail survey of 574 long-term rectal cancer survivors from Kaiser Permanente Northern California and Northwes was conducted in 2010-2011. The survey included questions on current depression and perceived financial burden from the City of Hope Quality of Life questionnaire. Responses were indicated on a scale where $0=$ 'none' and $10=$ 'severe'. Scores $\geq 4$ were classified as depressed and $\geq 5$ as high financial burden. ANOVA was used to contrast mean scores among surgery types. Linear regression models evaluated depression and perceived financial burden scores with co-variates. Results: Mean current depression level was 2.09 ( $\mathrm{SD}=2.40)$, with $23 \%$ (134 of 574) classified as depressed. PO survivors $(\mathrm{N}=182)$ had the highest current depression levels $(\mathrm{M}=2.46, \mathrm{SD}$ $=2.61)$ compared to $\mathrm{AN}(\mathrm{N}=326, \mathrm{M}=1.96, \mathrm{SD}=2.37)$ and $\mathrm{TO}(\mathrm{N}=66, \mathrm{M}$ $=1.68, \mathrm{SD}=1.81)(P=0.02)$. These differences in depression by surgery type were significant after adjusting for age, sex, and race $(P=0.015)$. Survivors reported average perceived financial burden of $1.94(\mathrm{SD}=2.72)$, with 18\% (103 of 572) perceiving high financial burdens. PO survivors perceived highest burden $(\mathrm{M}=2.5, \mathrm{SD}=2.92)$ compared to $\mathrm{AN}(\mathrm{M}=1.60$, $\mathrm{SD}=2.57)$ and $\mathrm{TO}(\mathrm{M}=2.12, \mathrm{SD}=2.65)(P=0.001)$. After confounding adjustment, level of perceived financial burden was positively associated with higher current depression levels $(P<0.001)$. Surgery type did not modify this relationship, but it was stronger among those also reporting depression after surgery $(P=0.04)$. Conclusions: Current depression was frequent among these long-term survivors of rectal cancer and was highest among those with permanent ostomy. While current depression and perceived financial burden due to their illness/treatment were statistically associated, there were no differences by surgery type. Research is needed to identify factors influencing high depression and financial pressures among these rectal cancer survivors. Clinicians should be aware that long-term survivors, particularly with permanent ostomy, can have depression and financial burdens resulting from their illness or treatment. Encouraging these survivors to receive psychosocial services may improve their well-being.

Keywords: Long-term rectal cancer survivors; Depression

doi:10.3121/cmr.2014.1250.ps2-39

PS2-42:

All-cause Mortality is Decreased in Women Undergoing Annual Mammography Screening Before Breast Cancer Diagnosis

Rachel Stankowski ${ }^{1}$; Jessica Engel ${ }^{2}$; Trista Stankowski-Drengler ${ }^{1}$; Hong Liang ${ }^{1}$; Adedayo Onitilo ${ }^{2}$

${ }^{1}$ Marshfield Clinic Research Foundation; ${ }^{2}$ Marshfield Clinic

Background/Aims: Annual mammogram screening for the early detection of breast cancer in women is recommended by most professional organizations beginning at the age of 40 . However, the efficacy of annual mammography screening related to decreasing mortality remains an area of debate due to concerns regarding risk versus benefit. The potential for harm as a result of over-diagnosis of cancers that may not have been clinically significant extends beyond the extensively analyzed breast cancer-specific mortality. Therefore, we sought to examine screening mammography frequency and all-cause mortality in breast cancer patients. Methods: Primary breast cancer cases diagnosed within the Marshfield Clinic system from 2002 through 2008 were identified for retrospective review. Patients diagnosed with breast cancer at another facility, male breast cancer patients, and patients residing outside of Wisconsin and its neighboring states were excluded. Patient demographics and additional data, including number of mammograms performed within five years prior to breast cancer diagnosis, breast cancer stage, insurance status, family history, and comorbidities, were abstracted. Kaplan-Meier curves were generated to assess the association between mammography screening in the five years prior to breast cancer diagnosis and overall survival with adjustment for age, insurance status, number of medical encounters, comorbidities, family history, and calendar year. Results: A total of 1,422 women with breast cancer were included in the analysis. After adjustment, women who missed any of their last five annual screening mammograms had a 2.3 -fold increase in all-cause mortality compared to subjects who participated in annual screening mammography. When subjects were stratified by the number of missed screening mammograms in the five years prior to breast cancer diagnosis, a progressive decline in survival was noted resulting in an all-cause mortality hazard ratio of 1.3 (95\% confidence interval $1.19-1.37, P<0.0001)$ for each additional missed mammogram. Conclusions: These results suggest that annual mammography prior to breast cancer diagnosis is predictive of increased overall survival. Importantly, a stepwise decline in overall survival was noted for each additional missed mammogram. These results are similar to findings for breast cancer-specific mortality and illustrate the importance of recommending annual screening mammography to all eligible women.

Keywords: Breast cancer; Mammograms

doi:10.3121/cmr.2014.1250.ps2-42

\section{Cardiovascular Disease}

PS1-40:

Preventable Major Cardiovascular Events Due to Uncontrolled Glucose, Blood Pressure, and Lipids or Active Smoking in Adults With Diabetes With and Without Cardiovascular Disease

Gabriela Vazquez Benitez'; Jay Desai ${ }^{1}$; Emily Schroeder ${ }^{2}$; Gregory Nichols ${ }^{3}$; Jodi Segal ${ }^{4}$; Andy Karter ${ }^{5}$; John Steiner ${ }^{2}$; Katherine Newton ${ }^{6}$; Leo Morales ${ }^{6}$; Ram Pathak ${ }^{7}$; Patrick O'Connor ${ }^{1}$

${ }^{1}$ HealthPartners; ${ }^{2}$ Kaiser Permanente Colorado; ${ }^{3}$ Kaiser Permanente Northwest; ${ }^{4} \mathrm{~J}$ ohns Hopkins University; ${ }^{5}$ Kaiser Permanente Northern California; ${ }^{6}$ Group Health Research Institute; ${ }^{7}$ Marshfield Clinic Research Foundation

Background/Aims: To analyze the incidence of major cardiovascular disease(CVD) hospitalization events and all-cause mortality among adults with diabetes with or without cardiovascular disease; and to estimate the proportion of preventable events due to uncontrolled lipids, glucose, blood pressure, and to active smoking. Methods: This was a retrospective cohort 